\title{
INTRODUCCIÓN: JUVENTUD E HISTORIA*
}

\author{
SANDRA SOUTO KuSTRÍN \\ Instituto de Historia del CSIC (España)
}

\begin{abstract}
"Año tras año se siente cada vez más entre la juventud que todo lo existente ha fracasado, que todas las consignas que se dan son un engaño y un fraude», "la juventud está harta de explicaciones, promesas y frases; lo que quiere es que suceda, por fin, algo decisivo» ${ }^{1}$.

«La edad de los «otros» importantes en relación con tu propia edad no es simplemente una cuestión de edad, sino de raza, nacionalidad, clase y estatus social, sexo, y un sinfín de otras variables. Pero el mundo es diferente para personas de diferente edad y generación incluso aunque tengan en común el género, la clase, la nacionalidad y la ocupación ${ }^{2}$.
\end{abstract}

Como indican las dos frases con que se inicia esta presentación, ni el creciente papel y la importancia cada vez mayor de los jóvenes en la Europa de entreguerras eran ignorados por los contemporáneos, ni la compleja interacción entre la edad y otras divisiones sociales fue negada por todos los científicos sociales. Pero ni la historiografía ha destacado lo primero hasta hace unas décadas, ni los sociólogos han abandonado una aproximación — fuertemente influi-

* La coordinación de este número monográfico ha sido posible gracias a una beca posdoctoral de la Comunidad de Madrid, a un contrato posdoctoral (I3P) del Consejo Superior de Investigaciones Científicas y a la participación en el proyecto de investigación «Grupos profesionales, corporativismo y políticas sectoriales del Estado durante la Dictadura de Primo de Rivera, 1923-1930» (HUM 200400406/HIST).

1 FISCHER, Ernst: Krise der Jugend, Viena-Leipzig, 1931, reproducido en FisCHER, Ernst: Problemas de la generación joven (entre la impotencia y la responsabilidad), Madrid, 1967, pp. 62 y 65.

2 Strauss, Anselm L.: Mirrors and Masks: The Search for Identity, Glencoe (Illinois), 1959, p. 138, cit. por Rintala, Marvin: "A generation in Politics: A Definition», en The Review of Politics (Notre Dame, In.), vol. 25, nº. 4 (octubre 1963), pp. 509-522, p. 509. 
da por interpretaciones puramente psicológicas de la adolescencia y por el funcionalismo parsoniano- que daba unas características únicas, comunes y universales a la adolescencia y a la juventud hasta prácticamente el mismo momento en que los historiadores descubrían a los jóvenes como un objeto de estudio muy importante para entender la edad contemporánea, cuando surge verdaderamente el fenómeno juvenil tal y como hoy lo conocemos.

La juventud, como periodo de la vida de una persona en el que la sociedad deja de verle como un niño pero no le da un estatus y funciones completos de adulto es, como todo grupo de edad, una construcción social e histórica, porque su significado, desarrollo, forma, contenido y duración dependen del orden económico, social, cultural y político de cada sociedad, de su localización histórica y del modo en que cada grupo es construido en una sociedad ${ }^{3}$. Aunque desde tiempos inmemoriales se ha hablado de juventud y se puede rastrear la existencia de grupos de jóvenes por consideraciones de edad desde las primeras civilizaciones de la Antigüedad, como Grecia y Roma ${ }^{4}$, y, especialmente, durante el Antiguo Régimen, la amplitud de sus límites cronológicos, su importancia numérica y, sobre todo, su papel en la sociedad y en la política - y la percepción de su importancia por diferentes instancias sociales y políticas, como gobiernos, partidos políticos, sindicatos o confesiones religiosas - no tenían nada que ver con los que alcanzarían con la modernización. Por ello, se tiende a considerar que la concepción de los jóvenes como grupo social definido y con características propias, en su definición actual, se desarrolló en Europa entre finales del siglo XVIII y principios del siglo XIX. Especialmente importante para esta evolución fueron las consecuencias de los cambios producidos por la modernización económica, social y política y el desarrollo del Estado moderno: las transformaciones de las relaciones laborales y de la forma de acceso a los oficios y profesiones o el aumento de la demanda de trabajadores no cualificados — normalmente jóvenes- como consecuencia de la industrialización; el establecimiento de un periodo de educación obligatoria que se fue ampliando con el paso del tiempo y que se hizo cada vez más importante para asegurar el acceso al trabajo y el mantenimiento del estatus social, al desaparecer la organización estamental de la sociedad; la creación de «ejércitos nacionales» a través del servicio militar obligatorio; la regulación del derecho de voto con el desarrollo de los sistemas liberalesdemocráticos; o el surgimiento de actividades de ocio que se dirigieron específi-

3 Wallace, Claire y Kovatcheva, Sijka: Youth in Society. The Construction and Deconstruction of Youth in East and Western Europe, Basingstoke, 1998, pp. 9 y 29; WIN, Johanna y WHITE, Robert, Rethinking Youth, Londres, 1997, pp. 9 y ss.; SolÉ I SERRATOSA, Joan: «Qui són els joves», en UCELAY DA CAL, Enric (Dir.): La joventut a Catalunya al segle XX. Materials per a una historia. I., Barcelona, 1987, pp. 38-55, p. 40.

4 HeER, Friedrich: Challenge of Youth, Londres, 1974, pp. 7-8. Un análisis muy interesante de la terminología utilizada por los griegos para hablar de la juventud en GANGUTIA ELíCEGUI, Elvira: «La fuerza y lo efímero. Jóvenes en los poemas homéricos», en MARíN, Manuela (coord.): Jóvenes en la historia, Mélanges de la Casa de Velázquez (Madrid), vol. 34-1 (primavera 2004), pp. 17-42, pp. 18-25. 
camente a un público juvenil. Aparecieron así toda una serie de instituciones y reglamentaciones que si bien, por una parte, aumentaron el periodo de dependencia de los jóvenes por consideraciones de edad, por otra, les dieron un perfil característico y facilitaron tanto su organización como su actuación de forma independiente. A pesar de que algunas de estas instituciones - como el ejército o la escuela - no eran nuevas, sí lo era su extensión a todos los estratos sociales. Por tanto, muchas de las «marcas» que fijan las fronteras contemporáneas entre niños, jóvenes y adultos no existían o estaban organizadas de forma diferente antes de lo que llamamos modernidad'.

Sin embargo, los jóvenes por consideraciones de edad no han formado nunca un todo homogéneo sino que han reflejado las divisiones económicas, sociales y políticas existentes en la sociedad. Además, el fenómeno que conocemos como modernización tuvo diferente ritmo y cronología en los distintos países. Todo esto hizo que el proceso de configuración de la juventud como grupo social tuviese también un ritmo distinto, no sólo en cada país, sino dentro de éstos, en el mundo urbano y en el rural, en las diferentes clases sociales y los géneros: la juventud surgió en primer lugar como un fenómeno urbano, masculino y de clases altas y medias ${ }^{6}$.

A lo largo de este proceso aparecieron asociaciones voluntarias, tanto dirigidas por los adultos como creadas de forma independiente por los mismos jóvenes, que organizaron a éstos en función de diferentes objetivos sociales, políticos, culturales o religiosos. Pero el origen y el papel de los llamados movimientos juveniles es muy variado: pueden ser el resultado de una organización sistemática y deliberada o pueden surgir de forma espontánea; pueden ayudar a integrar a la juventud en la sociedad y en el Estado, como muestran organizaciones tan dife-

5 Una completa y apretada síntesis de los cambios producidos por la modernización en la situación de la juventud, en LESLIE, W. Bruce: “Time, the subtle thief of youth": Historians and youth", en Youth and Policy. The Journal of Critical Análisis (Durham), nº 11 (invierno 1984/85), pp. 49-51. Véase también, entre una numerosa bibliografía, Wallace, Claire y Kovatcheva, Sijka: Youth in Society..., op. cit., pp. 11 y ss.; GILLIS, John Randall: Youth and History. Tradition and change in European Age Relations 1770-Present, Nueva York, San Francisco y Londres, 1974, capítulo 2, «Troubled youth: the consequences of Modernization, 1770-1870», pp. 37 y ss.; MitTERAUER, Michael: A bistory of youth, Oxford, 1992, pp. 125 y ss.; GRIFFIN, Christine: Representations of youth: the study of youth and adolescence in Britain and America, Cambridge, 1993, pp. 12 y ss.; o KRIEGEL, Annie: "Generational Differences: the History of an Idea», en Daedalus (Cambridge, Mass.), vol. $107, \mathrm{n}^{\circ} .4$ (otoño de 1978), pp. 23-38, pp. 23-26.

6 Bourdieu, Pierre: «La "jeunesse" n'est qu'un mot», en Bourdieu, Pierre: Questions de Sociologie, París, Les Editions de Minuit, 1980, pp. 143-154, passim; Wallace, Claire y Kovatcheva, Sijka: Youth in Society..., op. cit., pp. 34 y 49 y ss.; GILlIS, John Randall: Youth and History..., op. cit., pp. 75 y 125; MitTERAUER, Michael: A history of youth, op. cit., pp. 28 y 88; WIN, Johanna y WHITE, Robert: Rethinking Youth, op. cit., pp. 30 y 36; ROSENMAYR, Leopold: «Historico-comparative Sociology of Youth: The Case of Europe», en ROSENMAYR, Leopold y AllerbeCK, Klaus (eds.): "Youth and Society», en Current Sociology. La sociologie contemporaine. The Journal of the International Sociological Association (París), vol. 27, nº. 2/3 (1979), pp. 46-58, passim. 
rentes como los Boy Scouts, la Hitlerjugend o el Komsomol, o pueden intentar cambiar más o menos radicalmente la sociedad y/o el Estado7.

Fue en el periodo comprendido entre las dos guerras llamadas mundiales cuando los jóvenes se convirtieron en un problema social y político, pero también fueron considerados el origen del futuro, los transformadores de la sociedad, como no lo habían sido antes. La Primera Guerra Mundial y sus consecuencias no influyeron en todos los que vivieron sus años formativos durante 1914-1918 por igual, pero todos fueron decisivamente influidos por ella, incluso en los países - como España- que no participaron en el conflicto bélico, como se refleja en los artículos de este número. La crisis de 1929 dio un «último» empuje al llamado "problema juvenil»y/o «conflicto generacional», debido a que los jóvenes fueron uno de los sectores más afectados, no sólo porque se vieron especialmente golpeados por el aumento del desempleo como consecuencia de dicha crisis, sino porque las respuestas a esta última les afectaron también directamente: por ejemplo, las familias retiraron a sus hijos de los centros de enseñanza; los gobiernos redujeron los presupuestos destinados a la educación; y cientos de jóvenes de clase media vieron peligrar su futuro profesional. En todo el continente, los diferentes sectores sociales y políticos mostraron un interés cada vez mayor por la juventud; las publicaciones y los discursos dirigidos hacia ella se multiplicaron; se produjo una politización cada vez mayor de los jóvenes; sus organizaciones crecieron como nunca antes y la juventud jugó un papel destacado, e incluso protagonista, en la conflictividad del periodo y en el desarrollo de nuevos movimientos políticos, como el comunismo, el fascismo o el nazismo. De ahí que haya considerado en otro sitio este periodo como el de la primera «gran oleada» de movilización juvenil en el mundo occidental, en contraposición a la valoración tradicional que destacaba la «novedad» de los movimientos juveniles de los años sesenta del siglo $\mathrm{XX}^{8}$.

Esta aparición de la juventud como un grupo claramente definido y como un problema social explica que el concepto de adolescencia surgiera en el ámbito académico a principios del siglo XX con la obra de G. Stanley Hall; que las primeras aproximaciones sociológicas al concepto de juventud se elaboraran en

7 Colton, Joël: «Définition de la Jeunesse et des Mouvements de Jeunesse. La Jeunesse et la paix» en COMMISSION INTERNATIONALE D'HISTOIRE DES MOUVEMENTS SOCIAUX ET DES STRUCTURES SOCIALES: La jeunesse et ses mouvements. Influence sur l'évolution des sociétés aux XIXe et XXe siècles, París, 1992, pp. 3-14, pp. 7 y ss.; BraUngarT, Richard G. y BRAUnGART, Margaret M.: «Historical Generations and Citizenship: 200 years of youth movements», en Research in Political Sociology (Greenwich, Conn.), vol. 6 (1993), pp. 139-174, pp. 186-187; MitTERAUER, Michael: $A$ history of youth, op. cit., pp. 153 y ss.

8 GRESH, Levi D.: «Britain Tackled the Youth Problem», en The Journal of Educational Sociology (Nueva York), vol. 13, nº 6 (febrero de 1940), pp. 360-364; NeumanN, Sigmund: «The Conflict of Generations in Contemporary Europe: From Versailles to Munich», en Vital Speeches of the Day (Nueva York), vol V., no 20 (1 de agosto de 1939), pp. 623-628. Dogliani, Patrizia: Storia dei Giovani, Milán, 2003, pp. 65 y ss.; Souto Kustrín, Sandra: "El mundo ha llegado a ser consciente de su juventud como nunca antes": Juventud y movilización política en la Europa de entreguerras», en MARín, Manuela (coord.): Jóvenes en la historia, op. cit., pp. 179-215, p. 205. 
los años veinte de dicho siglo; o que fuera en el periodo previo a la Primera Guerra Mundial cuando José Ortega y Gasset abordó por primera vez, aunque muy brevemente, el tema de las generaciones, concepto que cobraría importancia y sobre el que se desarrollarían las principales teorías existentes - la del mismo Ortega pero también la de Karl Mannheim - en el periodo de entreguerras $^{9}$. A partir de las obras de ambos investigadores se han destacado la adolescencia y la juventud como periodos clave en la adquisición de una identidad propia por parte de las generaciones. Pero este concepto se ha utilizado en el análisis histórico, político y sociológico con diferente significado: relaciones de parentesco, cohorte o grupo de edad, y etapa de la vida, entre otros. En general, la mayoría de los estudios sobre el «conflicto generacional» hablan de las relaciones entre personas de diferentes grupos de edad, como se hace en los distintos artículos de este monográfico ${ }^{10}$.

Los enfoques sobre la juventud han evolucionado ligados a la situación histórica, al papel de los jóvenes en la sociedad y a la misma evolución de los movimientos juveniles y de las teorías predominantes en cada momento en las ciencias sociales. El desarrollo de los estudios sociológicos e históricos sobre la juventud se vio favorecido por las grandes movilizaciones estudiantiles de los años 60 del siglo XX. Pero desde el funcionalismo parsoniano - dominante en la sociología en las décadas centrales de esa centuria- sólo se podían interpretar las culturas y las protestas juveniles como resultado de situaciones de anomia, de falta de unas normas consistentes para dirigir la conducta, en suma, como una situación anormal. Ha sido, por tanto, desde las investigaciones empíricas — históricas o con una importante base histórica-, desde las que más se ha avanzado en el estudio del surgimiento y desarrollo de la juventud como grupo social. Se han ido introduciendo cada vez más dimensiones históricosociales, como el género o la raza, considerando que la juventud como fenóme-

9 Hall, G. Stanley: Adolescence: its psychology and its relations to physiology, anthropology, sociology, sex, crime, religion and education, Londres, 1904; MEAD, Margaret: Coming of Age in Samoa. A psychological study of primitive youth for Western civilisation, Nueva York, 1928; THRASHER, Frederick Milton: The Gang: a study of 1313 gangs in Chicago, Chicago (Illinois), 1927; ORTEGA Y GASSET, José: Vieja y nueva política (conferencia dada en el teatro de la Comedia el 23 de marzo de 1914), en Ibid: Obras Completas, vol. 1, Madrid, 1966, pp. 265-307; El tema de nuestro tiempo, Madrid, Espasa Calpe, 1988 (or. 1923); y En torno a Galileo. Esquema de la crisis, Madrid, 1965 (or. 1933); MANNHEIM, Karl: «El problema de las generaciones», en Revista Española de Investigaciones Sociológicas (Madrid), nº 62 (abril-junio 1993), pp. 193-242, pp. 216-217 (or. 1928).

10 Una síntesis en castellano de las distintas teorías de las generaciones en ARÓSTEGUI, Julio: La historia vivida. Sobre la historia del presente, Madrid, 2004, pp. 111-121; un análisis más amplio de sus problemas en JAEGER, Hans: "Generations in History: Reflections on a Controversial Concept», en History and Theory (Middletown, CT), vol. 24, n'. 3 (octubre, 1985), pp. 273-292. Las variadas aplicaciones del término generación se recogen, entre otras obras, en MAUGER, Gérard: «Élements pour une réflexion critique sur la catégorie de «jeunesse», Actes du colloque international Historicité de l'enfance et de la jeunesse, Athènes, 1-5 octobre 1984, Atenas, 1986, pp. 133-149, p. 146; o KERTZER, David I.: «Generation as a Sociological Problem», en Annual Review of Sociology (Palo Alto, California), vol. 9 (1983), pp. 125-149, pp. 133-134. 
no social depende, más que de la edad, de la posición de la persona dentro de varias estructuras sociales, entre las que destacan la familia, la escuela, el trabajo o las cohortes; y de las instituciones públicas estatales que con su legislación alteran la posición de los jóvenes en estos grupos ${ }^{11}$.

La historiografía sobre la juventud y sobre los movimientos juveniles en la edad contemporánea ha alcanzado un gran desarrollo en todo el continente europeo en las últimas décadas. De la mano de la historia social, pero también de la historia política o cultural, se han desarrollado numerosas investigaciones sobre el surgimiento y desarrollo de la juventud como grupo social, las diferentes culturas juveniles, su organización, o su participación en la conflictividad social y política. Son estudios en los que los jóvenes se analizan desde muy diversas perspectivas que, a veces, no se integran entre sí: hay investigaciones centradas en la educación, en la familia, en el ocio, en la delincuencia juvenil o en organizaciones específicas $^{12}$. Los análisis más abundantes son los referidos a Alemania, que acapara en gran medida la atención de la historiografía, no sólo por la importancia de la movilización de la juventud llevada a cabo por el nazismo, sino por la larga historia del movimiento juvenil alemán, cuyos orígenes se remontan a los años de la Confederación Germánica creada tras el Congreso de Viena de 1815. Pero, en general, en casi todos los países europeos hay ya un número importante de investigadores y de estudios dedicados a la problemática juvenil desde un punto de vista histórico, como se refleja en los artículos de este monográfico. Sin embargo, este desarrollo ni siquiera se vislumbra en España. Los análisis sobre la juventud, las políticas dirigidas hacia ella, sus formas de vida, sus organizaciones o su participación en la conflictividad social y política en el periodo anterior a los años 60 del siglo XX son escasos y no han tenido, hasta ahora, una gran continuidad $^{13}$. Además, frente a lo que sucede en otros países europeos y como pasa con otros temas históricos, prácticamente no hay estudios de investigadores españoles sobre la situación de la juventud y su organización en la Europa de entreguerras, mientras que las traducciones de obras de investigadores extranjeros sobre estas cuestiones son escasas, lo que hace que este fenómeno de escala continental sea prácticamente desconocido en España ${ }^{14}$.

11 Un análisis detallado de los diferentes planteamientos teóricos, sus limitaciones, y su relación con el contexto histórico y epistemológico del momento en SOUTO KuSTRÍN, Sandra: "Juventud, teoría e historia: la formación de un sujeto social y de un objeto de análisis», en Revista de Historia Actual (Cádiz), (en prensa).

12 Un breve estado de la cuestión de la historiografía sobre los movimientos juveniles en Europa en el periodo de entreguerras y sus limitaciones en SOUTO KUSTRíN, Sandra: «El mundo ha llegado...», op. cit., pp. 187-190.

13 Como se refleja en GonzÁlez Calleja, Eduardo y Souto Kustrín, Sandra: «Juventud y política en España: orientación bibliográfica», en Ayer (Madrid), n. 59-3 (2005), pp. 283-298.

14 Véanse, entre los más que escasos ejemplos sobre la Europa de entreguerras, MORENTE VALERO, Francisco, «Libro e moschetto», Barcelona, 2001; SÁEZ MARÍN, Juan, «Asociacionismo juvenil en Europa hasta 1940 (Notas para Estudio, I)», en De Juventud (Madrid), n . 5 (enero-marzo 1982), pp. 37-57; o SOUTO KuSTRÍN, Sandra, «"El mundo ha llegado...”, op. cit.; LEvi, Giovanni y 
Por todo esto, me plantee en este número monográfico ofrecer a los investigadores españoles una visión de conjunto de los estudios sobre la juventud en la Europa de entreguerras. Sugerí a diversos especialistas que tienen una importante trayectoria de investigación sobre diferentes aspectos de la problemática juvenil del periodo en cinco países que se pueden considerar clave en el entorno europeo occidental -Alemania, España, Francia, Italia y el Reino Unido-, que realizaran un análisis de la juventud en estos países en el periodo de entreguerras. La propuesta que se les hizo incluía un amplio conjunto de temas y cuestiones relacionados con la juventud, para que, sin voluntad de tratar de abarcar todos ellos exhaustivamente —algo imposible dada la extensión de un artículo- se mostraran las características más importantes de la situación de los jóvenes en los diferentes marcos políticos y culturales del continente europeo o alguno de los aspectos más relevantes de ésta. El resultado es un conjunto de estudios que recogen algunos de los temas más destacados de la problemática juvenil y de su investigación en estos países y que reflejan que, a pesar de las diferencias, había una serie de características y problemas comunes entre la juventud europea del periodo de entreguerras.

En primer lugar, el profesor Arnaud Baubérot pone en cuestión la novedad de los movimientos juveniles al analizar la relación de los existentes en la Francia del periodo de entreguerras con sus precedentes del Antiguo Régimen ${ }^{15}$. El estudio de un contexto nacional claramente pluriconfesional como el francés destaca el papel de las distintas confesiones religiosas en la creación y desarrollo de numerosas e importantes organizaciones juveniles de carácter cultural y/o educativo, tema que ha sido uno de los objetos centrales de interés de la investigación de este profesor. Pero también nos muestra, aunque sea más brevemente, que el predominio de estas organizaciones preferentemente culturales dio paso en el periodo de entreguerras a claros intentos de politización del movimiento juvenil: como dice el profesor Baubérot, «la inclinación de la juventud por el activismo y el radicalismo representaba entonces un valor que las diferentes fuerzas políticas trataban de captar y poner al servicio de sus intereses». La comparación de estos modernos movimientos juveniles con los grupos juveniles existentes en Francia durante el Antiguo Régimen nos indica que los primeros asumieron parte de las funciones de los segundos, como la manifestación de una cultura propia de la juventud o la defensa de los valores de la comunidad, pero tuvieron una mayor autonomía y responsabilidad, se ampliaron

SchmitT, Jean-Claude (eds.): Historia de los jóvenes, Madrid, 1996; KNOPP, Guido: Los niños de Hitler: retrato de una generación manipulada, Barcelona, 2001; y SCHOLL, Inge: La Rosa Blanca, Barcelona, 1994.

15 Frente a lo planteado, entre otros, por Richard G. Braungart («Historical Generations and Youth Movements: A Theoretical Perspective», en Research in Social Movements, Conflict and Change (Greenwich, Conn.), vol. VI, 1984, pp. 95-142, pp. 122 y ss.; e «Historical and Generational Patterns of Youth Movements: A Global Perspective», en Comparative Social Research (Greenwich, Conn.), vol. VII, 1984, pp. 3-62, p. 3); Joël Colton («Définition de la Jeunesse...», op. cit., passim); o Claire Wallace y Sijka Kovatcheva (Youth in Society..., op. cit., pp. 62 y ss.). 
a mujeres jóvenes y adolescentes, y llegaron a pasar de transmisores de los valores preponderantes en la sociedad a heraldos de una clase u organización.

Marco Fincardi y Elizabeth Harvey analizan los dos países europeos en los que - junto con la Rusia Soviética, la España franquista y el Portugal de Oliveira Salazar - el Estado intentó encuadrar desde arriba a la juventud. El fascismo italiano llevaría a cabo, como muestra Marco Fincardi, el encuadramiento autoritario de los jóvenes en una «juventud de Estado» y, por ser el primer país europeo en que esto se intentó, se convirtió en un modelo para otros. Frente a lo que se ha planteado tradicionalmente, el autor nos muestra que el fascismo no fue el primer movimiento que descubrió el valor de la movilización juvenil, y que tan o más importante que las formas de encuadramiento de los jóvenes en sí mismas, fue la adopción de una mística juvenil por parte del régimen fascista. Pero, como también analiza detalladamente el profesor Fincardi, este encuadramiento no soportaría la prueba de la Segunda Guerra Mundial y «en los primeros meses de 1943 fueron principalmente los fermentos juveniles los que atravesaron el país, disgregando la disciplina militar y los aparatos de control social».

Este fracaso del encuadramiento de los jóvenes en una «juventud de Estado» también se produjo en el Tercer Reich alemán. La situación especial de Alemania viene determinada en gran medida por el enorme desarrollo de los movimientos juveniles de todo tipo en el periodo previo a la subida de los nazis al poder, los llamados «imperios de la juventud» con que inicia la profesora Elizabeth Harvey su contribución a este número. En la Alemania de los años treinta la juventud «se construía como un problema y una causa potencial de desorden» pero, al mismo tiempo, como "potencial vanguardia de un nuevo orden». La cuanto menos particular relación entre muchas de las organizaciones juveniles y el régimen nacionalsocialista, las causas de su compromiso con dicho régimen en sus fases iniciales y las consecuencias de esta estrategia son otros de los elementos centrales de este artículo, pero la doctora Harvey concluye también que este intento de adoctrinamiento que buscaba crear una juventud conformista fracasó y dio lugar a distintas formas de resistencia y rebelión.

Al igual que en el caso italiano, estos actos de disidencia se relacionan con lo que James Scott llamó, analizando movimientos campesinos en el Tercer Mundo, las «armas de los pobres», en cierta medida la resistencia más relevante, porque es la que se imbrica dentro de la vida cotidiana. Y aunque no se puede sobrestimar el papel de estas formas de resistencia, tampoco se debe subestimarla: la resistencia «organizada» no logró acabar con los regímenes fascistas italiano y alemán, que sólo cayeron por la acción de las potencias aliadas. Además, los estudios sobre la protesta social, como el del mismo Scott, han mostrado la importancia de todo acto de disidencia, por pequeño que sea, para el mantenimiento o desarrollo de tradiciones, conceptos culturales o ideologías en periodos de represión ${ }^{16}$.

16 ScOTT, James C.: Weapons of the Weak: everyday forms of peasant resistance, New Haven y Londres, 1985. 
El artículo de Eduardo González Calleja y Sandra Souto Kustrín muestra el retraso con el que se desarrollaron los movimientos juveniles en España, pero también que, a pesar de estar poco estudiada, la movilización juvenil tiene ya una larga historia que, como en otros países de nuestro entorno, tuvo su origen en asociaciones estudiantiles universitarias. Analiza el desarrollo y la consolidación de las organizaciones juveniles en España durante la dictadura de Primo de Rivera y la Segunda República, cuando pasaron de ser organismos dependientes de sus referentes adultos a buscar - y lograr en muchos casos- cotas cada vez mayores de autonomía e independencia: evolucionaron, usando la terminología de Abrams, de «juventud en sí» a «juventud para sí» ${ }^{17}$, y dieron lugar a un periodo caracterizado por «la implicación extrema en la vida política» de la juventud, que tuvo su culminación durante la guerra civil, en la que se destaca el papel y la importancia que se le dio a los jóvenes en ambos bandos en conflicto.

Si todos los artículos de este monográfico muestran que sería más correcto hablar de juventudes que de juventud, dado que, como ya se ha planteado, ésta ha reflejado siempre las divisiones económicas, sociales, de género, culturales, políticas y regionales existentes, el trabajo de la profesora Selina Todd lo destaca de forma especial, estableciendo claramente las diferentes posibilidades de acceso a la educación, al trabajo y a las actividades de ocio en la Inglaterra de entreguerras — en un periodo en que la oferta de estas últimas se estaba incrementando constantemente y estaba dirigida principalmente hacia los jóvenes. Muestra, además, la complicada interacción entre género y clase, introduciéndonos en el mundo de la mujer joven que, tradicionalmente, ha estado bastante marginada en los estudios sobre la juventud ${ }^{18}$. Pero también nos indica que, a pesar de las definiciones negativas de la adolescencia y la juventud establecidas desde estudios psicológicos como el de Hall citado anteriormente, o de la idea de un enfrentamiento generacional casi inevitable de las diferentes teorías de las generaciones, la realidad histórica es siempre mucho más compleja: los jóvenes trabajadores jugaban, en muchos casos, un papel fundamental en la economía familiar y la reciprocidad económica y emocional caracterizó las relaciones entre padres e hijos en la Inglaterra de entreguerras: «las imágenes populares de la juventud como un periodo de ocio e irresponsabilidad no representan con exactitud los estilos de vida de los trabajadores jóvenes».

Por tanto, estos artículos, en su unidad y en su diversidad, muestran la importancia de la juventud en el periodo analizado, pero también la variedad temática y metodológica y la riqueza de los estudios que se están realizando sobre ella. Prueban que más allá de afirmaciones generalistas y universales o

17 ABrams, Philip: «Rites de Passage. The Conflict of Generations in Industrial Society», en Laqueur, Walter y Mosse, George L. (eds.): «Generations in Conflict», en Journal of Contemporary History (Londres), vol. 5, nº. 1 (1970), pp. 175-190, pp. 186-187.

18 Como dice Michael Mitterauer, cuando se habla del pasado, «nuestro entendimiento de los jóvenes es concebido generalmente (...) en términos fuertemente masculinos» (MITTERAUER, Michael: $A$ bistory of youth, op. cit., p. 19). 
monocausales, el marco para entender la juventud debe incluir la continuidad y el cambio, las relaciones dentro y entre los diferentes grupos de edad, las representaciones que se asocian con ellos, y las divisiones sociales de clase, género, raza y/o etnia, en un proceso en el que los jóvenes se interrelacionan con muchas instituciones, como la escuela, la familia, la Iglesia o el Estado. Buscan también abrir el camino para la continuación —en cierta medida, el iniciode los estudios sobre la juventud y sus movimientos en España y ayudar a la realización de análisis comparativos con fenómenos similares del ámbito europeo, del cual el estudio de la historia de España no se puede separar, y que probablemente nos muestren, como esta sucediendo en muchas otras cuestiones historiográficas, que nuestra historia no es tan peculiar como algunas veces se nos ha querido hacer creer.

Para concluir hay que decir que todo trabajo de este tipo supone contraer numerosas deudas. En primer lugar, con los mismos colaboradores, que acogieron con excepcional interés la realización de este monográfico. En segundo lugar, con el comité de redacción de la revista Hispania, tanto con el que aprobó la publicación de este dossier, —y especialmente con su director, Francisco Villacorta Baños - por la acogida de esta propuesta y los ánimos recibidos para llevarla a cabo; como con el comité de redacción actual, por el trabajo dedicado a su publicación, y, especialmente, a su directora, Isabel Alfonso, por sus sugerencias. Por último, pero no menos importante, quisiera destacar la ayuda recibida de Eduardo González Calleja, Martin Johnson y Jill Parsons para hacer frente a las complicaciones producidas por la elaboración de un número monográfico con textos escritos originalmente en diferentes lenguas. A todos ellos vaya, desde aquí, mi mayor agradecimiento. 